\title{
Oral Antidiabetics and Sleep Among Type 2 Diabetes Patients: Data From the UK Biobank
}

\author{
Pei Xue ${ }^{1,2}$, Jiafei $W u^{1}$, Xiangdong Tang $^{2}$, Xiao $\operatorname{Tan}^{1,3 \dagger}$ and Christian Benedict ${ }^{1 \text { *t }}$ \\ ${ }^{1}$ Department of Neuroscience (Sleep Science, Biomedicinskt centrum (BMC)), Uppsala University, Uppsala, Sweden, ${ }^{2}$ Sleep \\ Medicine Center, Department of Respiratory and Critical Care Medicine, Mental Health Center, Translational Neuroscience \\ Center, and State Key Laboratory of Biotherapy, West China Hospital, Sichuan University, Chengdu, China, ${ }^{3}$ Department of \\ Clinical Neuroscience, Karolinska Institutet, Solna, Sweden
}

\section{OPEN ACCESS \\ Edited by:}

Alexandra Kautzky-Willer, Medical University of Vienna, Austria

Reviewed by: Mohammad Qaddoumi, Kuwait University, Kuwait Shinichi Oikawa, Nippon Medical School, Japan

*Correspondence:

Christian Benedict christian.benedict@neuro.uu.se

${ }^{\text {t}}$ These authors have contributed equally to this work

Specialty section:

This article was submitted to Clinical Diabetes, a section of the journal Frontiers in Endocrinology

Received: 23 August 2021 Accepted: 14 October 2021 Published: 03 November 2021

Citation:

Xue $P$, Wu J, Tang $X$, Tan $X$ and Benedict C (2021) Oral Antidiabetics and Sleep Among Type 2 Diabetes Patients: Data From the UK Biobank.

Front. Endocrinol. 12:763138. doi: 10.3389/fendo.2021.763138
Previous small-scale studies have found that oral antidiabetic therapy is associated with sleep difficulties among patients with type 2 diabetes (T2D). Here, we used data from 11806 T2D patients from the UK Biobank baseline investigation to examine the association of oral antidiabetic therapy with self-reported difficulty falling and staying asleep and daily sleep duration. As shown by logistic regression adjusted for, e.g., age, T2D duration, and $\mathrm{HbA} \mathrm{A}_{1 \mathrm{c}}$, patients on non-metformin therapy ( $\mathrm{N}=815 ; 86 \%$ were treated with sulphonylureas) had a 1.24-fold higher odds ratio of reporting regular difficulty falling and staying asleep at night compared to those without antidiabetic medication use $(\mathrm{N}=5366, \mathrm{P}<0.05)$ or those on metformin monotherapy ( $\mathrm{N}=5625, \mathrm{P}<0.05)$. Non-metformin patients reported about 8 to 10 minutes longer daily sleep duration than the other groups $(P<0.05)$. We did not find significant differences in sleep outcomes between untreated and metformin patients. Our findings suggest that non-metformin therapy may result in sleep initiation and maintenance difficulties, accompanied by a small but significant sleep extension. The results of the present study must be replicated in future studies using objective measures of sleep duration and validated questionnaires for insomnia. Considering that most T2D patients utilize multiple therapies to manage their glycemic control in the long term, it may also be worth investigating possible interactions of antidiabetic drugs on sleep.

Keywords: type 2 diabetes, metformin, sulphonylurea, insomnia, sleep duration, UK Biobank

\section{INTRODUCTION}

Type 2 diabetes (T2D) patients often complain about sleep difficulties (1). For instance, about 77\% regularly report insomnia symptoms, such as difficulty falling and staying asleep (2). According to the International Classification of Sleep Disorders criteria (3), having trouble falling asleep at night and waking up in the middle of the night constitute key insomnia symptoms. In addition to insomnia, T2D patients often report habitual short (usually defined as $<7$ hours per day) or long sleep duration (often described as $>9$ hours per day) (4).

Several factors may account for sleep difficulties among T2D patients, including but not limited to comorbidities such as overweight and hypertension (1). As suggested by previous studies, the type of oral antidiabetic agent used to prevent hyperglycemia may also account for sleep difficulties among 
T2D patients (5-7). For example, by using one-night sleep polysomnography, a study among 387 outpatients with T2D found that those on metformin slept on average about 36 minutes longer and exhibited a $6.4 \%$ higher sleep efficiency compared to patients on non-metformin (mainly sulfonylureas) (5). Metformin, a biguanide, suppresses hepatic glucose production and intestinal glucose absorption and promotes $\beta$ cell functions and insulin sensitivity (8-10). Sulfonylureas lower blood glucose by stimulating insulin release from pancreatic $\beta$ cells $(11,12)$.

Besides the mechanism of action, oral antidiabetics can also differ in the type, frequency, and severity of side effects (13-19). For instance, hypoglycemia represents the most common side effect of sulphonylureas (17). Diarrhea, nausea, vomiting, abdominal bloating, abdominal cramping or pain, flatulence, and anorexia, occur in approximately $10 \%$ to $30 \%$ of metformin recipients (18, 19). Side effects caused by oral antidiabetic therapy may lead to sleep difficulties, primarily if occurring during sleep.

In the present study, we wanted to examine the association of oral antidiabetics with sleep among T2D patients from the UK Biobank. Specifically, we used cross-sectional data from 11806 T2D patients to determine whether the ability to initiate or maintain sleep and habitual sleep duration would vary by the type of oral antidiabetic agent used.

\section{MATERIALS AND METHODS}

\section{Study Design and Participants}

From 2006 to 2010, the UK Biobank recruited about 500000 individuals. For the present study, we used the two following criteria to identify T2D patients: (a) we used a validated algorithm based on self-reported disease, medication, and T2D diagnosis in medical history (20); and (b) the patient used glucoselowering medication and had an hemoglobin $\mathrm{A}_{1 \mathrm{c}}\left(\mathrm{HbA}_{1 \mathrm{c}}\right)$ level $\geq 6.5 \%$ ( $48 \mathrm{mmol} / \mathrm{mol})$. Records on antihyperglycemic medication derived from the UK Biobank interview. We assigned patients to one of the following three groups: not treated with oral antidiabetics, treated with metformin, and being on non-metformin therapy. We did not consider patients eligible for the analysis when they were on two or more oral antidiabetics (e.g., metformin and sulphonylurea) or insulin therapy. We also excluded individuals with probable type 1 diabetes or gestational diabetes identified by the diabetes algorithm (20). Following further exclusions as detailed in Table 1, 11806 T2D patients represented our final sample.

The UK Biobank study was approved by the National Health Service National Research Ethics Service (ref. 11/NW/0382) and all participants provided written informed consent to participate in the UK Biobank study. Information about ethics oversight in the UK Biobank can be found at https://www.ukbiobank.ac.uk/ethics/.

\section{Outcomes}

Habitual sleep duration was estimated by the question "About how many hours sleep do you get every 24 hours? (please include naps)". Patients were also asked, "Do you have trouble falling asleep at night or do you wake up in the middle of the night?". They had the following response options: "never/rarely", "sometimes," and "usually." For the logistic regression analysis, the response option "usually" was defined as an event, i.e., the patients often complained about having trouble falling asleep at night or waking up in the middle of the night.

\section{Confounders}

Confounders were selected based on our a priori knowledge of the relationships among potential confounders, intermediate variables, and outcome variables. Age (continuous scale), sex, region of UK Biobank assessment center, Townsend deprivation index reflecting a person's socioeconomic status (continuous scale), BMI (continuous scale), and systolic blood pressure (continuous scale) derived from the UK Biobank reception information, baseline characteristics, touchscreen questionnaire, or physical measurement. Obstructive sleep apnea (OSA) represents common comorbidity of T2D (21), which can affect both sleep quality and duration (22). One of OSA's cardinal symptoms is snoring (22). Thus, in the present study, we controlled for selfreported snoring. Snoring was assessed by the following question: "Does your partner or a close relative or friend complain about your snoring?" Patients had the four following response options: "Yes," "No," "Don't know," or "Prefer not to answer." For the analysis, we excluded those responding, "Don't know" or "Prefer not to answer" (Table 1). The duration of T2D (continuous scale) was calculated by subtracting self-reported age at T2D diagnosis from the age when the participant attended the UK Biobank baseline investigation. We also controlled our analysis for the use of antidepressants (selective serotonin reuptake inhibitors, serotonin-norepinephrine reuptake inhibitors, and tricyclic antidepressants) and statins, as both can influence sleep $(23,24)$. In addition, we also retrieved data on the use of sedatives, such as barbiturates, benzodiazepines, Z-drugs, antihistamines, and other sedative medications. Finally, information about T2D comorbidities and complications was retrieved from the UK Biobank interview. Due to the low prevalence of sedative use, stroke, chronic kidney disease, and T2D complications, these variables were not considered as confounders in the logistic regression analysis.

Findings from previous studies suggest that glycemic control, often assessed by the $\mathrm{HbA}_{1 \mathrm{c}}$, is inversely associated with sleep

TABLE 1 | Exclusions.

\begin{tabular}{lc}
\hline & $\begin{array}{c}\text { Number of } \\
\text { subjects }\end{array}$ \\
\hline Initial cohort & 502543 \\
T2D patients & 27370 \\
Treated with two or more oral antidiabetic medications & 5626 \\
Treated with insulin & 3131 \\
No information regarding insomnia (dependent variable) & 95 \\
No information regarding sleep duration (dependent variable) & 240 \\
No information regarding snoring (confounder) & 1591 \\
No information regarding BMl (confounder) & 132 \\
No information regarding systolic blood pressure (confounder) & 35 \\
No information regarding HbA Ac $_{1 \mathrm{c}}$ (confounder) & 1024 \\
No information regarding diabetes duration (confounder) & 3669 \\
No information regarding Townsend index (confounder) & 21 \\
Final cohort & $\mathbf{1 1 ~ 8 0 6}$
\end{tabular}

T2D, type 2 diabetes; BMI, body mass index; $\mathrm{HbA}_{1 \mathrm{c}}$, hemoglobin $\mathrm{A}_{1 \mathrm{c}}$. 
quality and duration (25-27). Thus, we also controlled our analysis for patients' $\mathrm{HbA}_{1 \mathrm{c}}$. The $\mathrm{HbA}_{1 \mathrm{c}}$ (continuous scale) was determined with high-performance liquid chromatography using the Bio-Rad VARIANT II TURBO $\mathrm{HbA}_{1 \mathrm{c}}$ analyzer.

\section{Statistical Analysis}

We performed all analyses with IBM SPSS version 24.0 (Inc., Chicago, IL, USA). Baseline characteristics among the three treatment groups were compared with univariate generalized linear models and chi-square tests. We used logistic regression analysis to compare the odds ratio of having trouble falling asleep at night or waking up in the middle of the night among the three groups. Finally, we explored possible group differences in daily sleep duration by generalized linear models. Overall, a p-value smaller than 0.05 was considered significant.

\section{RESULTS}

Baseline characteristics are summarized in Table 2. In the full cohort $(n=11806), \sim 35 \%$ of the patients reported that they usually struggle with falling or staying asleep. As shown in the fully-adjusted logistic regression (Figure 1), patients using nonmetformin agents $(n=815)$ had a 1.24-fold higher odds ratio of reporting regular difficulty falling and staying asleep at night than patients without antidiabetic medication use $(n=5366$; $\mathrm{P}=0.007)$. Additional multivariable analysis revealed that patients using non-metformin had higher odds of having trouble falling and staying asleep when using metformin patients as the reference group ( $\mathrm{n}=5$ 625) (OR[95\%-CI], 1.244 $[1.064,1.455] ; \mathrm{P}=0.006)$. Non-metformin patients also reported longer sleep duration than untreated and metformin patients ( $\mathrm{P}=0.004$ for the main effect of treatment group; derived from the fully-adjusted generalized linear model; see Figure $\mathbf{2}$ for group comparisons). Comparisons between metformin patients and those without antidiabetic medication use did not reveal significant group differences (Figures 1, 2).

\section{DISCUSSION}

There is evidence that oral antidiabetics may impact sleep; however, with mixed results. One study reported longer and more efficient sleep among T2D patients on metformin than

TABLE 2 | Baseline characteristics of untreated, metformin, and non-metformin patients with type 2 diabetes.

\begin{tabular}{|c|c|c|c|c|}
\hline Characteristic & \multicolumn{3}{|c|}{ Oral antidiabetic regimen } & $P$ value \\
\hline Age at investigation, mean (SD), y & $60.7(6.6)$ & $59.8(6.9)$ & $61.3(6.7)$ & $<.001^{\dagger}$ \\
\hline Difficulty falling and staying asleep & $1843(34.3)$ & $1936(34.4)$ & $310(38.0)$ & $.106^{\#}$ \\
\hline Sleep duration, mean (SD), h & $7.19(1.29)$ & $7.19(1.34)$ & $7.37(1.45)$ & $.001^{\dagger}$ \\
\hline Snoring & $2573(48.0)$ & $2635(46.8)$ & $365(44.8)$ & $.182^{\#}$ \\
\hline $\mathrm{HbA1c}$, mean (SD), mmol/mol & $45.8(10.2)$ & $51.6(11.7)$ & $53.2(13.2)$ & $<.001^{\dagger}$ \\
\hline Duration of type 2 diabetes, mean (SD), y & $4.6(7.6)$ & $5.7(7.9)$ & $8.3(9.0)$ & $<.001^{\dagger}$ \\
\hline \multicolumn{5}{|l|}{ Region of assessment center } \\
\hline England & $4696(87.5)$ & $5091(90.5)$ & 695 (85.3) & \multirow[t]{3}{*}{$<.001^{\#}$} \\
\hline Wals & $283(5.3)$ & $214(3.8)$ & $32(3.9)$ & \\
\hline Scotland & $387(7.2)$ & $320(5.7)$ & $88(10.8)$ & \\
\hline \multicolumn{5}{|l|}{ Type 2 diabetes comorbidities } \\
\hline CVD $(120,121,125, \mid 48 \text { and } 150)^{\star}$ & $102(1.9)$ & $113(2.0)$ & $28(3.4)$ & $.015^{\#}$ \\
\hline Stroke $(160,161,163,164)^{\star}$ & $12(0.2)$ & $16(0.3)$ & $6(0.7)$ & - \\
\hline Chronic kidney Disease (N03, N11, N18)* & $2(0.04)$ & 0 & $1(0.1)$ & - \\
\hline Type 2 diabetes complication & $3(0.1)$ & $13(0.2)$ & $3(0.4)$ & - \\
\hline \multicolumn{5}{|l|}{ Type of oral antidiabetic monotherapy } \\
\hline Metformin & - & 100 & - & - \\
\hline Sulfonylurea & - & - & 85.9 & - \\
\hline Glitazone & _- & _- & 12.8 & - \\
\hline Meglitinide & _- & _- & 1.2 & - \\
\hline Other oral antidiabetics & _- & _- & 0.1 & - \\
\hline
\end{tabular}

$B M I$, body mass index; $H b A_{1 c}$, hemoglobin $A_{1 c} ; C V D$, cardiovascular disease.

Data are presented as number (percentage) of study participants unless otherwise indicated.

${ }^{\dagger}$ Univariate generalized linear model.

${ }^{*}$ Chi-square tests.

${ }^{*}$ Referring to $I C D-10$ codes. 


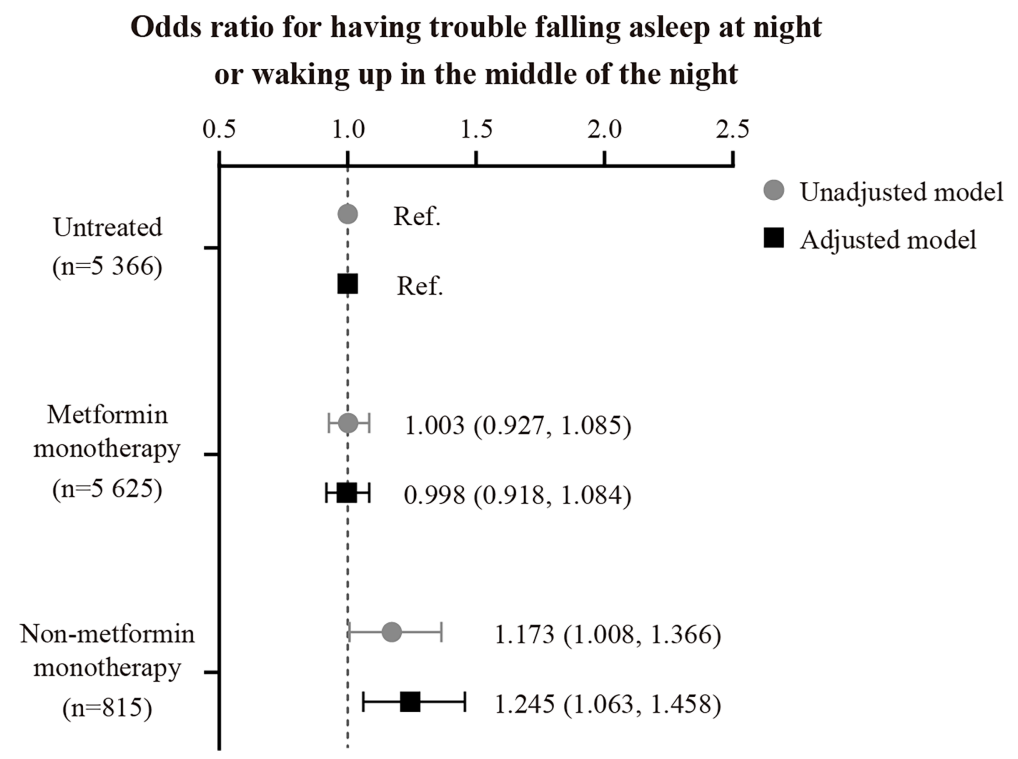

FIGURE 1 | Unadjusted and adjusted odds ratios and 95\% Cls for having trouble falling asleep at night or waking up in the middle of the night, stratified by the oral antidiabetic regimen. The adjusted model was controlled for patients' age, sex, BMI, UK Biobank assessment center, Townsend index, systolic blood pressure, snoring status, type 2 diabetes duration, $\mathrm{HbA}_{1 \mathrm{c}}$, presence of cardiovascular disease, antidepressant therapy status, and statin therapy status.

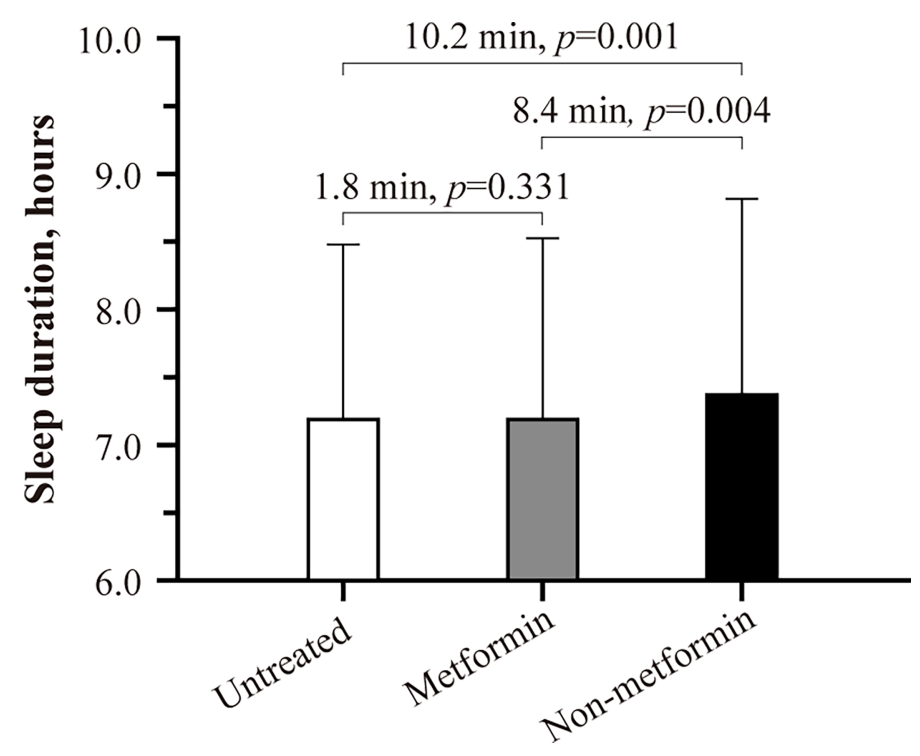

FIGURE 2 | Habitual sleep duration of untreated, metformin, and non-metformin patients with type 2 diabetes. Estimated marginal means \pm SE derived from a generalized linear model adjusting for patients' age, sex, BMI, UK Biobank assessment center, Townsend index, systolic blood pressure, snoring status, type 2 diabetes duration, $\mathrm{HbA}_{1 \mathrm{c}}$, presence of cardiovascular disease, antidepressant therapy status, and statin therapy status are shown. $\mathrm{P}$ smaller than 0.05 was considered significant.

those using non-metformin agents (5). In contrast, two other studies found that metformin may be associated with poor sleep quality and frequent nightmares $(6,7)$. In the present study, including 11806 patients with T2D, we found that those treated with non-metformin were more likely to report difficulty falling and staying asleep at night compared to those without antidiabetic medication use or those on metformin monotherapy. More than four out of five patients in the non-metformin group were on sulfonylurea monotherapy. Hypoglycemia, the most common side effect of sulphonylureas (17), can induce counterregulatory stress 
hormone responses (28). If occurring at bedtime or during sleep, these hypoglycemia-induced endocrine stress responses may cause hyperarousal and, therefore, impair the ability of the patient to fall and stay asleep. However, due to the lack of hypoglycemia screening in the UK Biobank, this explanation remains speculative. We also found that patients on non-metformin slept about 8 to 10 minutes longer than those on metformin or without antidiabetic treatment. Whether the small sleep extension was due to accompanying sleep initiation and maintenance difficulties among non-metformin patients is unclear.

Several limitations apply to the present study. It remains unclear whether factors such as antidiabetic dosing and timing of medication administration may modulate the association of oral antidiabetics with sleep. Furthermore, the UK Biobank investigation did not survey adverse side effects of oral antidiabetics, which could explain differences in sleep outcomes between metformin and non-metformin users. In T2D patients with low kidney function, physicians often prescribe sulfonylureas (29). Chronic kidney disease has been linked to poor sleep quality (30). Importantly, only three of 11 806 patients had chronic kidney disease at the baseline investigation in the present study. Thus, it is unlikely that the observed sleep difficulties among patients treated with nonmetformin are attributable to chronic kidney disease. Furthermore, in the present study, only a few patients reported the use of sleep-promoting sedatives. However, we could not control our analysis for the possible use of sleep-promoting overthe-counter-remedies (e.g., melatonin). The present study was further limited by the small sample size of patients treated with non-metformin drugs. Thus, future studies with larger sample sizes, including novel types of non-metformin oral antidiabetics than those investigated herein (e.g., dipeptidyl-peptidase 4 inhibitors, glucagon-like peptide-1 receptor agonists), are needed to advance our understanding of how oral antidiabetics impact sleep among patients with T2D. Considering that most T2D patients utilize multiple therapies to manage their glycemic control in the long term, it may also be worth investigating possible interactions of antidiabetic drugs on sleep. Finally, sleep duration was derived from self-reports, which might be subject to potential recall bias. Thus, our results must be replicated in studies using longitudinal measures of sleep duration (e.g., sleeptracking wearables) and validated questionnaires for insomnia (e.g., insomnia severity index).

Despite these limitations, the primary strength of our study is that our results were based on one of the largest cohorts worldwide. Furthermore, findings were robust to adjustment for important confounders, such as snoring, age, BMI, blood pressure, and $\mathrm{HbA}_{1 \mathrm{c}}$.

\section{CONCLUSIONS}

Recurrent problems with falling and staying asleep have been associated with impaired glycemic control among patients with T2D (31-33). To mitigate these possible adverse consequences,
T2D patients on non-metformin should regularly participate in sleep screenings. As shown herein, they more often suffer from sleep initiation and maintenance difficulties.

\section{DATA AVAILABILITY STATEMENT}

The datasets presented in this article are not readily available because data may be obtained from a third party and are not publicly available. Data were derived from the UK Biobank investigation. Thus, data may be obtained from the UK Biobank upon request. Requests to access the datasets should be directed to https://www.ukbiobank.ac.uk/.

\section{ETHICS STATEMENT}

The studies involving human participants were reviewed and approved by National Health Service National Research Ethics Service (ref. 11/NW/0382). The patients/participants provided their written informed consent to participate in this study.

\section{AUTHOR CONTRIBUTIONS}

$\mathrm{CB}$ and PX designed the study. PX and JW performed analysis. $\mathrm{CB}$ and PX drafted the manuscript. All authors interpreted the results and critically revised the manuscript for intellectual content. PX takes responsibility for the accuracy of the data analysis. All authors approved the final version of this manuscript to be published.

\section{FUNDING}

The authors' work is funded by the Novo Nordisk Foundation (CB; NNF19OC0056777), Swedish Brain Research Foundation (CB; FO2020-0044), Åke Wiberg Foundation (XiaoT; M18-0169, M19-0266), Fredrik and Ingrid Thuring Foundation (XiaoT; 2019-00488), and the Swedish Society for Medical Research (XiaoT). The study sponsor/funder was not involved in the design of the study; the collection, analysis, and interpretation of data; writing the report; and did not impose any restrictions regarding the publication of the report.

\section{ACKNOWLEDGMENTS}

This research has been conducted using the UK Biobank resource under project number 55177. 


\section{REFERENCES}

1. Tan X, van Egmond L, Chapman CD, Cedernaes J, Benedict C. Aiding Sleep in Type 2 Diabetes: Therapeutic Considerations. Lancet Diabetes Endocrinol (2018) 6:60-8. doi: 10.1016/S2213-8587(17)30233-4

2. Gupta S, Wang Z. Predictors of Sleep Disorders Among Patients With Type 2 Diabetes Mellitus. Diabetes Metab Syndr (2016) 10:213-20. doi: 10.1016/j.dsx. 2016.06.009

3. American Academy of Sleep Medicine and International classification of sleep disorders (3rd edn). (2014).

4. Shan Z, Ma H, Xie M, Yan P, Guo Y, Bao W, et al. Sleep Duration and Risk of Type 2 Diabetes: A Meta-Analysis of Prospective Studies. Diabetes Care (2015) 38:529-37. doi: 10.2337/dc14-2073

5. Kajbaf F, Fendri S, Basille-Fantinato A, Diouf M, Rose D, Jounieaux V, et al. The Relationship Between Metformin Therapy and Sleep Quantity and Quality in Patients With Type 2 Diabetes Referred for Potential Sleep Disorders. Diabetes Med (2014) 31:577-80. doi: 10.1111/dme.12362

6. Façanha C, Bruin V, Bruin P, Façanha A, Rocha HC, Araujo M, et al. Hyperglycemia in Pregnancy: Sleep Alterations, Comorbidities and Pharmacotherapy. Rev Assoc Med Bras (1992) 2021) 67:45-51. doi: 10.1590/ 1806-9282.67.01.20200216

7. Yanto TA, Huang I, Kosasih FN, Lugito NPH. Nightmare and Abnormal Dreams: Rare Side Effects of Metformin? Case Rep Endocrinol (2018) 17:2018. doi: 10.1155/2018/7809305. 7809305.

8. Viollet B, Guigas B, Sanz Garcia N, Leclerc J, Foretz M, Andreelli F. Cellular and Molecular Mechanisms of Metformin: An Overview. Clin Sci (Lond) (2012) 122:253-70. doi: 10.1042/CS20110386

9. Rojas LB, Gomes MB. Metformin: An Old But Still the Best Treatment for Type 2 Diabetes. Diabetol Metab Syndr (2013) 5:6. doi: 10.1186/1758-5996-5-6

10. Radziuk J, Bailey CJ, Wiernsperger NF, Yudkin JS. Metformin and its Liver Targets in the Treatment of Type 2 Diabetes. Curr Drug Targets Immune Endocr Metabol Disord (2003) 3:151-69. doi: 10.2174/1568008033340298

11. Lv W, Wang X, Xu Q, Lu W. Mechanisms and Characteristics of Sulfonylureas and Glinides. Curr Top Med Chem (2020) 20:37-56. doi: 10.2174/ 1568026620666191224141617

12. Proks P, Reimann F, Green N, Gribble F, Ashcroft F. Sulfonylurea Stimulation of Insulin Secretion. Diabetes (2002) 51(Suppl 3):S368-76. doi: 10.2337/ diabetes.51.2007.s368

13. Maruthur NM, Tseng E, Hutfless S, Wilson LM, Suarez-Cuervo C, Berger Z, et al. Diabetes Medications as Monotherapy or Metformin-Based Combination Therapy for Type 2 Diabetes: A Systematic Review and MetaAnalysis. Ann Intern Med (2016) 164:740-51. doi: 10.7326/M15-2650

14. Drugscom. Metformin Side Effects (2020). Available at: https://www.drugs. $\mathrm{com} / \mathrm{sfx} / \mathrm{metformin}$-side-effects.html.

15. Pawlyk AC, Giacomini KM, McKeon C, Shuldiner AR, Florez JC. Metformin Pharmacogenomics: Current Status and Future Directions. Diabetes (2014) 63:2590-9. doi: 10.2337/db13-1367

16. Diabetes Treatment: Medications for Type 2 Diabetes (2020). Available at: https://www.mayoclinic.org/diseases-conditions/type-2-diabetes/indepth/ diabetes-treatment/art-20051004.

17. Schloot NC, Haupt A, Schütt M, Badenhoop K, Laimer M, Nicolay C, et al. Risk of Severe Hypoglycemia in Sulfonylurea-Treated Patients From Diabetes Centers in Germany/Austria: How Big is the Problem? Which Patients are at Risk? Diabetes Metab Res Rev (2016) 32:316-24. doi: 10.1002/dmrr.2722

18. Blonde L, Dailey GE, Jabbour SA, Reasner CA, Mills DJ. Gastrointestinal Tolerability of Extended-Release Metformin Tablets Compared to ImmediateRelease Metformin Tablets: Results of a Retrospective Cohort Study. Curr Med Res Opin (2004) 20:565-72. doi: 10.1185/030079904125003278

19. Hoffmann IS, Roa M, Torrico F, Cubeddu LX. Ondansetron and MetforminInduced Gastrointestinal Side Effects. Am J Ther (2003) 10:447-51. doi: 10.1097/00045391-200311000-00012

20. Eastwood SV, Mathur R, Atkinson M, Brophy S, Sudlow C, Flaig R, et al. Algorithms for the Capture and Adjudication of Prevalent and Incident
Diabetes in UK Biobank. PloS One (2016) 11(9):e0162388. doi: 10.1371/ journal.pone. 0162388

21. Tasali E, Mokhlesi B, Van Cauter E. Obstructive Sleep Apnea and Type 2 Diabetes: Interacting Epidemics. Chest (2008) 133:496-506. doi: 10.1378/ chest.07-0828

22. Ralls F, Cutchen L. A Contemporary Review of Obstructive Sleep Apnea. Curr Opin Pulm Med (2019) 25:578-93. doi: 10.1097/MCP.0000000000000623

23. Mayers AG, Baldwin DS. Antidepressants and Their Effect on Sleep. Hum Psychopharmacol (2005) 20:533-59. doi: 10.1002/hup.726

24. Szmyd B, Rogut M, Białasiewicz P, Gabryelska A. The Impact of Glucocorticoids and Statins on Sleep Quality. Sleep Med Rev (2021) 55:101380. doi: 10.1016/j.smrv.2020.101380

25. Tan X, Benedict C. Sleep Characteristics and Hbalc in Patients With Type 2 Diabetes on Glucose-Lowering Medication. BMJ Open Diabetes Res Care (2020) 8:e001702. doi: 10.1136/bmjdrc-2020-001702

26. Mokhlesi B, Temple KA, Tjaden AH, Edelstein SL, Utzschneider KM, Nadeau KJ, et al. Association of Self-Reported Sleep and Circadian Measures With Glycemia in Adults With Prediabetes or Recently Diagnosed Untreated Type 2 Diabetes. Diabetes Care (2019) 42:1326-32. doi: $10.2337 / \mathrm{dc} 19-0298$

27. Brouwer A, van Raalte DH, Rutters F, Elders PJM, Snoek FJ, Beekman ATF, et al. Sleep and Hbalc in Patients With Type 2 Diabetes: Which Sleep Characteristics Matter Most? Diabetes Care (2020) 43:235-43. doi: 10.2337/ dc19-0550

28. Cryer PE. Glucose Counterregulation: Prevention and Correction of Hypoglycemia in Humans. Am J Physiol (1993) 264:E149-55. doi: 10.1152/ ajpendo.1993.264.2.E149

29. Eppenga WL, Lalmohamed A, Geerts AF, Derijks HJ, Wensing M, Egberts A, et al. Risk of Lactic Acidosis or Elevated Lactate Concentrations in Metformin Users With Renal Impairment: A Population-Based Cohort Study. Diabetes Care (2014) 37:2218-24. doi: 10.2337/dc13-3023

30. Natale P, Ruospo M, Saglimbene VM, Palmer SC, Strippoli GF. Interventions for Improving Sleep Quality in People With Chronic Kidney Disease. Cochrane Database Syst Rev (2019) 5:CD012625. doi: $10.1002 / 14651858$

31. Ding C, Zhang J, Lau ESH, Luk AOY, So WY, Ma RCW, et al. Gender Differences in the Associations Between Insomnia and Glycemic Control in Patients With Type 2 Diabetes: A Cross-Sectional Study. Sleep (2019) 42: zsz014. doi: 10.1093/sleep/zsz014

32. Green MJ, Espie CA, Popham F, Robertson T, Benzeval M. Insomnia Symptoms as a Cause of Type 2 Diabetes Incidence: A 20 Year Cohort Study. BMC Psychiatry (2017) 17(1):94. doi: 10.1186/s12888-017-1268-4

33. Koopman ADM, Beulens JW, Dijkstra T, Pouwer F, Bremmer MA, van Straten A, et al. Prevalence of Insomnia (Symptoms) in T2D and Association With Metabolic Parameters and Glycemic Control: Meta-Analysis. J Clin Endocrinol Metab (2020) 105:614-43. doi: 10.1210/clinem/dgz065

Conflict of Interest: The authors declare that the research was conducted in the absence of any commercial or financial relationships that could be construed as a potential conflict of interest.

Publisher's Note: All claims expressed in this article are solely those of the authors and do not necessarily represent those of their affiliated organizations, or those of the publisher, the editors and the reviewers. Any product that may be evaluated in this article, or claim that may be made by its manufacturer, is not guaranteed or endorsed by the publisher.

Copyright $\odot 2021$ Xue, Wu, Tang, Tan and Benedict. This is an open-access article distributed under the terms of the Creative Commons Attribution License (CC BY). The use, distribution or reproduction in other forums is permitted, provided the original author(s) and the copyright owner(s) are credited and that the original publication in this journal is cited, in accordance with accepted academic practice. No use, distribution or reproduction is permitted which does not comply with these terms. 